\title{
Optimización de factores fisicoquímicos en la biodegradación de cianuro por Klebsiella sp. ART1, en biorreactor aireado
}

\author{
Optimization of physicochemical factors in cyanide biodegradation by Klebsiella \\ sp.ART1, in aerated biorreactor
}

\author{
Ana Beatriz Copari Mamani \\ 2," Milena Carpio Mamani \\ ORCID: 0000-0002-2090-4694 \\ César Julio Cáceda Quiroz \\ ORCID: 0000-0002-1405-6924 \\ ORDID: 0000-0002-8822-5954
}

\section{RESUMEN}

El cianuro es una sustancia altamente tóxica que representa un problema para el ambiente. Además es letal para muchos organismos vivos. El objetivo fue optimizar parámetros fisicoquímicos de $\mathrm{pH}$, temperatura y cianuro libre $\left[\mathrm{CN}^{-}\right]$en el proceso de biodegradación de cianuro por Klebsiella sp. ART1, en condiciones alcalinas y en biorreactores aireados, utilizando la metodología superficie respuesta (MSR). El modelo se ajustó mediante el diseño de Box- Behnken. La medición de cianuro libre y carga microbiana se realizaron cada 12 horas y la producción de amonio cada 24 horas. Además, se monitoreó constantemente el $\mathrm{pH}$ por un periodo de evaluación de 108 horas. Se obtuvo una tolerancia de $400 \mathrm{ppm}$ de cianuro libre con un inóculo inicial de $2 \times 10^{8}$ cél $/ \mathrm{ml}$, alcanzando una eficiencia de biodegradación del $98 \%$ en 60 horas de evaluación, el mismo que fue confirmado con el crecimiento bacteriano y con la producción de amonio. En el proceso de optimización solo el $\mathrm{pH}$ y [CN] fueron estadísticamente significativos. Después del ajuste del modelo, se determinó que los parámetros óptimos de degradación de cianuro en condiciones alcalina fueron a una temperatura de $30^{\circ} \mathrm{C}, \mathrm{pH} 10$ y $[\mathrm{CN}] 250 \mathrm{ppm}$, logrando una eficiencia de biodegradación de cianuro del $99 \%$. Se concluyó que Klebsiella sp. ART1 fue capaz de degradar cianuro en productos menos tóxicos; esto sugiere que podría ser utilizada como una alternativa de tratamiento en procesos de biorremediación.

Palabras clave: Biodegradación, Cianuro, Optimización, Klebsiella sp. ART1.

\section{ABSTRACT}

Cyanide is a highly toxic substance that represents a problem for the environment, it is also lethal for many living organisms. The objective was to optimize physicochemical parameters of $\mathrm{pH}$, temperature and free cyanide [CN-] in the cyanide biodegradation process by Klebsiella sp. ART1 in alkaline conditions and in aerated bioreactors using the surface response (RSM) methodology, the model was adjusted using the Box-Behnken design. The measurement of free cyanide, microbial load was carried out every 12 hours and the production of ammonia every 24 hours, in addition the $\mathrm{pH}$ was constantly monitored for an evaluation period of 108 hours. A tolerance of 400 ppm of free cyanide was obtained with an initial inoculum of $2 \times 108$ cells / $\mathrm{ml}$ that reached a $98 \%$ free cyanide biodegradation efficiency in 60 hours of evaluation that was confirmed with bacterial growth and ammonium production. In the optimization process, only the $\mathrm{pH}$ and [CN-] were statistically significant. After the model adjustment, it was determined that the optimal parameters of cyanide degradation in alkaline conditions were at a temperature of $30^{\circ} \mathrm{C}, \mathrm{pH} 10$ and [CN-] $250 \mathrm{ppm}$, achieving a cyanide biodegradation efficiency of $99 \%$. It was concluded that Klebsiella sp. ART1 was able to degrade cyanide in less toxic products; this suggests that it could be used as a treatment alternative in bioremediation processes.

Keywords: Biodegradation, Cyanide, Optimization, Klebsiella sp. ART1.

\footnotetext{
1,2,3 Laboratorio de Biorremediación, Facultad de Ciencias, Universidad Nacional Jorge Basadre Grohmann, 23000, Tacna-Perú.

* Autor de correspondencia: anelim.877@gmail.com
} 


\section{INTRODUCCIÓN}

El cianuro es usado en diversos procesos industriales del aluminio y acero (Gessner et al., 2005; Mondal et al., 2019), en la agricultura, en la producción de químicos orgánicos, plásticos, pinturas e insecticidas (Nyamunda, 2017), en la industria minera y joyería. También está presente en los residuos de galvanoplastía (LuqueAlmagro et al., 2016), generando grandes volúmenes de aguas residuales que contienen cianuro proveniente de las industrias. Representan un riesgo para muchas formas de vida, debido a que el cianuro es un compuesto altamente tóxico, siendo el cianuro de hidrógeno $(\mathrm{HCN})$ y el cianuro libre $\left(\mathrm{CN}^{-}\right)$los más tóxicos (Lottermoser, 2010; Ram, 2010). Todos los residuos líquidos cianurados deben contar con un tratamiento óptimo para minimizar el riesgo de contaminación por cianuro para el ambiente, la salud de la vida silvestre y los seres humanos (Luque-Almagro et al., 2016; Lovasoa et al., 2017). Para ello, una posible alternativa es el tratamiento biológico, que a través de la biodegradación resulta ser eficaz, confiable, ecológico y económicamente viable, siendo más rentables que los tratamientos químicos (Dash et al., 2009; Lovasoa et al., 2017). Además, podría ser utilizado con éxito en operaciones a gran escala (Ackil, 2003).

A pesar de la alta toxicidad de estos compuestos cianurados, existen microorganismos capaces de transformar el cianuro a compuestos menos tóxicos. Algunos de estos lo utilizan como fuente de carbono y/o nitrógeno para su crecimiento, que mediante diferentes enzimas, lo convierten en amoníaco y carbonato (Karamba et., 2015; Kumar et al., 2017) a través de diferentes rutas metabólicas (Gupta et al., 2010, Luque-Almagro et al., 2018). Algunas bacterias como Pseudomonas pseudoalcaligenes (LuqueAlmagro et al., 2011), Pseudomonas fluorescens (Kunz et al., 1992; Dursun et al., 2000), Bacillus sp. (Javaheri et al., 2017), Klebsiella oxytoca (Chen et al., 2008), hongos como Fusarium oxysporum (Akinpelu et al., 2017) y algas como Scenedesmus obliquus (Lovasoa et al., 2017), se han utilizado en la degradación de cianuro. Sin embargo, no solo se debe tener en cuenta las condiciones de degradación del compuesto; sino también, las condiciones óptimas en el que se desarrolla el microorganismo, debido a que los procesos pueden alterarse por la composición del medio, concentración inicial de cianuro, materia orgánica, $\mathrm{pH}$, adición de sustratos, temperatura y condiciones de cultivo. En el caso de biorremediación de agua, las condiciones óptimas de temperatura oscilan entre 25 a $50{ }^{\circ} \mathrm{C}$; pero la mayoría está alrededor de $30^{\circ} \mathrm{C}$ para bacterias y $43^{\circ} \mathrm{C}$ para hongos. El valor de $\mathrm{pH}$ oscila entre 5.2 a 10.5 para bacterias y 6 a 8.5 para hongos (Lovasoa et al., 2017).

Se ha determinado los parámetros de optimización de degradación de cianuro para Bacillus, Rodococcus, Serratia marcescens, Pseudomonas (Karamba et al., 2015). Para Klebsiella sp. solo se conoce que presenta una excelente biodegradación de cianuro entre 25 a 35 ${ }^{\circ} \mathrm{C}$, en concentraciones iniciales de cianuro de 100 a $500 \mathrm{ppm}$ y que crece a un $\mathrm{pH}$ neutro y alcalino (Kao et al., 2006; Chen et al., 2009; Khamar et al., 2015; Razanamahandry et al., 2016). Sin embargo, no se han reportado las condiciones óptimas de degradación en condiciones alcalinas, ni la interacción de estos parámetros en el proceso de biodegradación (Kumar et al., 2017; Lovasoa et al., 2017). Por lo tanto, es importante la optimización en los procesos biotecnológicos para lograr una óptima degradación (Karamba et al., 2015). Además, cada mejora en el proceso puede resultar en un aumento significativo en el rendimiento de la producción y ahorro en el costo de producción (Chen et al., 2005). También podría mejorar el crecimiento y proliferación de un microorganismo para someterlo a adecuadas manipulaciones biotecnológicas (Karamba et al., 2015). De este modo, la optimización puede ayudar a comprender el poder predictivo de lo que se puede esperar de los seres vivos incorporados a diseños (Jhonson, 2013).

El objetivo de la investigación fue optimizar parámetros fisicoquímicos de $\mathrm{pH}$, temperatura y cianuro libre $\left[\mathrm{CN}^{-}\right]$en el proceso de biodegradación de cianuro por Klebsiella sp. ART1, en condiciones alcalinas y en biorreactores aireados, utilizando la metodología superficie respuesta (MSR) y el modelo se ajustó mediante el diseño de Box- Behnken (Box \& Behnken, 1960; Mongomery, 2004).

\section{MATERIALY MÉTODOS}

\section{Caracterización del microorganismo}

La cepa de Klebsiella sp. ART1 fue aislada de las lagunas de estabilización de Magollo, TacnaPerú. Para la reactivación de la cepa, se sembró en caldo nutritivo, incubado a $35{ }^{\circ} \mathrm{C}$ por 24 horas. Luego, se sembró en medio sólido de agar nutritivo y agar Mac Conkey incubado a $35^{\circ} \mathrm{C}$ por 
24 horas. Para la caracterización y visualización de la cepa, se realizó tinción diferencial Gram, tinción de cápsula (Erick et al. 2012) y pruebas bioquímicas de rojo de metilo, citrato de Simmons, Voges Proskauer, Indol, TSI y LIA (Garrity et al., 2010) para su confirmación.

\section{Biodegradación de cianuro por Klebsiella sp.} ART1

Para la adaptación de la bacteria se utilizó medio líquido mineral $9 \mathrm{M}$ que contenía $(\mathrm{g} / \mathrm{L})$ : $\mathrm{Na}_{2} \mathrm{HPO}_{4} \cdot 7 \mathrm{H}_{2} \mathrm{O}(12.8) ; \mathrm{KH}_{2} \mathrm{PO}_{4}$ (3); $\mathrm{NaCl}(0.5)$; $\mathrm{MgSO}_{4} \cdot 7 \mathrm{H}_{2} \mathrm{O}(0.5) ; \mathrm{CaCl}_{2}(0.1)$; acetato de sodio $0.2 \%(\mathrm{w} / \mathrm{v})$ como fuente de carbono, extracto de levadura $0.2 \%(\mathrm{w} / \mathrm{v})$ como fuente de nitrógeno; y $1 \%(\mathrm{v} / \mathrm{v})$ de sales minerales que contenía $(\mathrm{g} / \mathrm{L})$ : $\mathrm{ZnSO}_{4} .7 \mathrm{H}_{2} \mathrm{O}(0.05) ; \mathrm{MnCl}_{2} .4 \mathrm{H}_{2} \mathrm{O}(0.05)$; $\mathrm{CuCl}_{2} .2 \mathrm{H}_{2} \mathrm{O}(0.005) ; \mathrm{Na}_{2} \mathrm{MoO}_{4} \cdot 2 \mathrm{H}_{2} \mathrm{O}$ (0.005); $\mathrm{Na}_{2} \mathrm{~B}_{4} \mathrm{O}_{7} \cdot 10 \mathrm{H}_{2} \mathrm{O}$ (0.002); $\mathrm{CoCl}_{2} \cdot 6 \mathrm{H}_{2} \mathrm{O}$ (0.0003). Además, se ajustó el pH a 10.5 (Huertas et al., 2010, Khamar et al., 2015) y se esterilizó a $121^{\circ} \mathrm{C}$ y 15 libras de presión por 15 minutos. El pH del medio se ajustó utilizando $\mathrm{NaOH} 2 \mathrm{M}$ o $\mathrm{HCl} 1 \mathrm{M}$. La cepa fue inoculada en medio $9 \mathrm{M}$ con extracto de levadura y con diferentes concentraciones de cianuro, iniciando de 50 a $500 \mathrm{ppm}$ de $\left[\mathrm{CN}^{-}\right]$y pH 10.5 (para evitar la volatilización del cianuro en forma de gas $\mathrm{HCN}$ ). Luego se incubó a $30^{\circ} \mathrm{C}$ por 24 h. Posteriormente, se inoculó la cepa en medio líquido $9 \mathrm{M}$ sin extracto de levadura y se incrementó la concentración de cianuro de $50-$ $500 \mathrm{ppm}^{\mathrm{de}}\left[\mathrm{CN}^{-}\right]$en un $\mathrm{pH} 10.5$, incubado también a $30^{\circ} \mathrm{C}$ por $24 \mathrm{~h}$ (Khamar et al., 2015). La producción de inóculo se realizó en 2 matraces que contenían $250 \mathrm{ml}$ de medio $9 \mathrm{M}$ con extracto de levadura y $500 \mathrm{ppm}$ de [CN], pH 10.5 e incubado a $30^{\circ} \mathrm{C}$ hasta llegar a una concentración inicial de $1.1 \times 10^{8}$ cél $/ \mathrm{ml}$. El análisis de crecimiento bacteriano se analizó mediante conteo en una cámara Petroff Hausser.

\section{Optimización de las condiciones de degradación de cianuro utilizando MSR}

La optimización se realizó según MSR, mediante el diseño de Box Behnken basado en bloques incompletos. Se utilizó para identificar los factores claves en la biodegradación de cianuro (Tabla 1), a partir de los cuales se determinaron los niveles óptimos de las variables significativas y los efectos de sus interacciones mutuas. Las variables independientes fueron la temperatura $\left(25,30\right.$ y $\left.35^{\circ} \mathrm{C}\right), \mathrm{pH}(10,10.5$ y 11$)$, concentración de cianuro libre $\left[\mathrm{CN}^{-}\right](200,300$ y $400 \mathrm{ppm})$ y la variable respuesta fue la eficiencia de biodegradación de cianuro por Klebsiella sp. ART1. Según el diseño, se realizaron 16 operaciones (Tabla 2) que fueron monitoreados por un periodo de evaluación de 108 horas.

Tabla 1. Variables incluidas en los experimentos de Box Behnken y niveles de concentración

\begin{tabular}{lcccc}
\multicolumn{1}{r}{ Variable } & Escala & $\begin{array}{c}\text { Nivel } \\
\text { Mínimo }\end{array}$ & $\begin{array}{c}\text { Nivel } \\
\text { Medio }\end{array}$ & $\begin{array}{c}\text { Nivel } \\
\text { Máximo }\end{array}$ \\
\hline $\begin{array}{l}\text { Temperatura } \\
\left({ }^{\circ} \mathrm{C}\right)\end{array}$ & $\mathrm{X}_{1}$ & 25 & 30 & 35 \\
$\mathrm{pH}$ & $\mathrm{X}_{2}$ & 10 & 10.5 & 11 \\
$\begin{array}{l}\text { Concentración } \\
\text { de }[\mathrm{CN}-\mathrm{ppm})\end{array}$ & $\mathrm{X}_{3}$ & 200 & 300 & 400 \\
\hline
\end{tabular}

Tabla 2. Diseño Box-Behnken usando tres variables

\begin{tabular}{ccccccc}
\hline & \multicolumn{6}{c}{ Variables } \\
\cline { 2 - 7 } $\mathbf{N}^{\circ}$ & & & & & [CN] \\
& $\mathbf{X}_{\mathbf{1}}$ & $\mathbf{X}_{\mathbf{2}}$ & $\mathbf{X}_{\mathbf{3}}$ & $\mathbf{T}\left(\mathbf{~}^{\mathbf{C}} \mathbf{C}\right)$ & $\mathbf{p H}$ & $\mathbf{p p m}$ \\
\hline 1 & -1 & -1 & 0 & 25 & 10 & 300 \\
2 & 1 & -1 & 0 & 35 & 10 & 300 \\
3 & -1 & 1 & 0 & 25 & 11 & 300 \\
4 & 1 & 1 & 0 & 35 & 11 & 300 \\
5 & -1 & 0 & -1 & 25 & 10.5 & 200 \\
6 & 1 & 0 & -1 & 35 & 10.5 & 200 \\
7 & -1 & 0 & 1 & 25 & 10.5 & 400 \\
8 & 1 & 0 & 1 & 35 & 10.5 & 400 \\
9 & 0 & -1 & -1 & 30 & 10 & 200 \\
10 & 0 & 1 & -1 & 30 & 11 & 200 \\
11 & 0 & -1 & 1 & 30 & 10 & 400 \\
12 & 0 & 1 & 1 & 30 & 11 & 400 \\
13 & 0 & 0 & 0 & 30 & 10.5 & 300 \\
14 & 0 & 0 & 0 & 30 & 10.5 & 300 \\
15 & 0 & 0 & 0 & 30 & 10.5 & 300 \\
16 & 0 & 0 & 0 & 30 & 10.5 & 300 \\
\hline
\end{tabular}

Nota: $\mathrm{X}_{1}, \mathrm{X}_{2}, \mathrm{X}_{3}$ representa el nivel codificado

La ecuación de regresión para este modelo es:

$$
\begin{aligned}
Y=a_{o}+a_{1} T & +a_{2} p H+a_{3}\left[C N^{-}\right]+a_{4} T^{2} \\
& +a_{5} p H^{2}+a_{6}\left[C N^{-}\right]^{2} \\
& +a_{7} T p H+a_{8} T\left[C N^{-}\right] \\
& +a_{9} p H\left[C N^{-}\right]
\end{aligned}
$$

Ec. 1

Donde:

$\mathrm{Y}=$ La degradación final de cianuro

$a=$ coeficiente del modelo

$T, p H,\left[C N^{-}\right]=$variables 


\section{Determinación de cianuro libre y amonio}

El cianuro libre se determinó con el método titulométrico (Estándar Methods 4500-CN-D. EEUU). Para cuantificar la concentración de cianuro libre se utilizó una proporción propuesta por Carrillo (2000). El método se basa en la reacción del $\mathrm{AgNO}_{3}$ con el ión cianuro en solución alcalina para formar el complejo soluble $\mathrm{Ag}(\mathrm{CN})_{2}$. Se utilizó $10 \mathrm{ml}$ de muestra y se agregó 3 gotas de Ioduro de potasio IK. Posteriormente, se tituló con $\mathrm{AgNO}_{3}$. La proporción para obtener la concentración de cianuro libre fue:

\section{$1 \mathrm{ml}$ de gasto de $\mathrm{AgNO}_{3}=20 \mathrm{ppm}$ de $\mathrm{CN}^{-}$}

Para determinar la concentración de amonio, se utilizó el método colorimétrico (Merck) que indica la cantidad de nitrógeno amónico $\left(\mathrm{NH}_{4}-\mathrm{N}\right)$, que se presenta en forma de iones amonio y amoniaco.

\section{Determinación de la eficiencia de biodegradación de cianuro}

La eficiencia de degradación (ED) de la bacteria degradadora de cianuro se calculó con la siguiente fórmula (Kandasamy et al., 2015):

Donde:

$$
D E(\%)=\left(\frac{I_{C}-F_{C}}{I_{C}}\right) \times 100
$$

$I_{C}=$ Concentración inicial de cianuro $(\mathrm{mg} / \mathrm{L})$

$R_{c}=$ Concentración final de cianuro $(\mathrm{mg} / \mathrm{L})$

\section{Análisis de datos}

Los datos se analizaron utilizando un análisis de varianza (ANOVA), para establecer si existe diferencia significativa entre los tratamientos para la máxima biodegradación de cianuro. El modelo se ajustó mediante el diseño Box Behnken. Los análisis de datos se realizaron utilizando el paquete estadístico Statistica v. 13.0.

\section{RESULTADOS}

\section{Crecimiento de la bacteria en presencia de cianuro}

La colonia de Klebsiella sp. ART1 en agar nutritivo era de color blanco cremoso, cóncavas, lisas y de borde circular bien definido (Fig. 1a). Por otro lado, en medio Mac Conkey (Fig. 1b), fueron colonias plano convexas, de borde ondulado, de aspecto mucoide y brillante. La cepa era un bacilo Gram negativo con cápsula y según las pruebas bioquímicas (Tabla 3), se confirma que corresponde a Klebsiella sp. ART1. Para la adaptación, se cultivó la cepa en medio mínimo $9 \mathrm{M}$ con extracto de levadura, y posteriormente en medio $9 \mathrm{M}$ con cianuro hasta llegar a $400 \mathrm{ppm}$ de $\left[\mathrm{CN}^{-}\right]$. La cepa fue capaz de degradar el cianuro, lo que indica que tuvo la capacidad de utilizar este compuesto como única fuente de nitrógeno, la adición de una mínima cantidad de extracto de levadura facilitó el crecimiento de la bacteria.

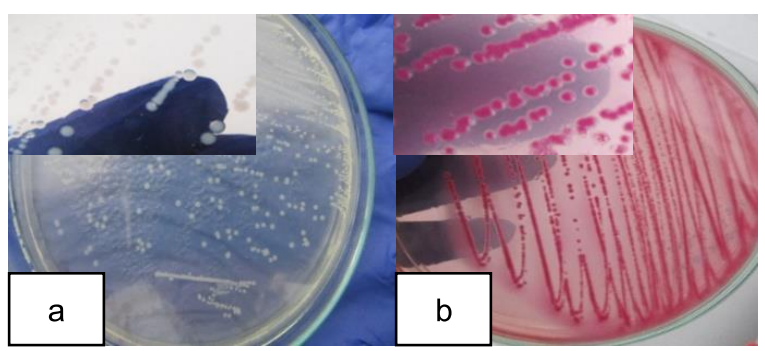

Figura 1. Crecimiento de Klebsiella sp. ART1: a) en Agar nutritivo y b) en Mac Conkey

Tabla 3. Caracterización bioquímica de Klebsiellasp. ART1

\begin{tabular}{cc}
\hline Bioquímica & $\begin{array}{c}\text { Klebsiella } \text { sp. } \\
\text { ART1 }\end{array}$ \\
\hline TSI & $\mathrm{A} / \mathrm{A}(\mathrm{g})$, \\
\hline LIA & $\mathrm{K} / \mathrm{K}$ \\
\hline Citrato & + \\
\hline Indol & + \\
\hline VP & + \\
\hline RM & - \\
\hline
\end{tabular}

Determinación de la eficiencia de degradación de cianuro

El efecto de la biodegradación de cianuro se realizó agregando 200, 300 y 400 ppm de cianuro al medio 9M, a diferentes condiciones de temperatura y $\mathrm{pH}$. Los resultados indican que en la mayoría de tratamientos, Klebsiella sp. ART1 obtuvo una alta eficiencia de biodegradación, logrando eliminar más del $98 \%$ de cianuro en 108 horas. Sin embargo, la mayor degradación se obtuvo a las 60 horas (Tabla 4). En el tratamiento 9 fue donde se tuvo la mayor tasa de biodegradación (98\%). La producción de amonio fue incrementándose de 0 a $1 \mathrm{mg} \mathrm{NH}_{4}^{+} / \mathrm{L}$ durante la evaluación. Esto indicaría que, mientras aumenta la producción de amonio, disminuye la cantidad de cianuro; y por lo tanto aumenta la eficiencia de degradación. 
Tabla 4. Biodegradación experimental de cianuro

\begin{tabular}{ccc}
\hline Tratamiento & $\begin{array}{c}{[\mathbf{C N}-] \text { res. }(\mathbf{p p m})} \\
\mathbf{6 0 h}\end{array}$ & $\begin{array}{c}\text { [CN-] exp. Biode. } \\
\mathbf{( \% )} * \mathbf{6 0 h}\end{array}$ \\
\hline $\mathbf{1}$ & 50 & 83.33 \\
$\mathbf{2}$ & 16 & 94.67 \\
$\mathbf{3}$ & 144 & 52.00 \\
$\mathbf{4}$ & 122 & 59.33 \\
$\mathbf{5}$ & 28 & 86.00 \\
$\mathbf{6}$ & 68 & 66.00 \\
$\mathbf{7}$ & 176 & 56.00 \\
$\mathbf{8}$ & 204 & 49.00 \\
$\mathbf{9}$ & 4 & 98.00 \\
$\mathbf{1 0}$ & 28 & 86.00 \\
$\mathbf{1 1}$ & 24 & 94.00 \\
$\mathbf{1 2}$ & 202 & 49.50 \\
$\mathbf{1 3}$ & 62 & 79.33 \\
$\mathbf{1 4}$ & 56 & 81.33 \\
$\mathbf{1 5}$ & 66 & 78.00 \\
$\mathbf{1 6}$ & 58 & 80.67 \\
\hline
\end{tabular}

Tabla 5. Análisis de varianza de la tasa de biodegradación de cianuro libre por Klebsiella sp. ART1

\begin{tabular}{cccccc}
\hline Fuente & $\boldsymbol{S C}$ & $\boldsymbol{d} \boldsymbol{f}$ & $\boldsymbol{C M}$ & $\boldsymbol{R a z o ́ n - F}$ & Valor-P \\
\hline A: T & 8.67 & 1 & 8.674 & 3.9699 & 0.1403 \\
B: $\mathrm{pH}$ & 1896.36 & 1 & 1896.356 & 867.9671 & $0.0001^{*}$ \\
$\mathrm{C}:$ [CN-] & 957.03 & 1 & 957.031 & 438.0357 & $0.0002^{*}$ \\
AA & 631.27 & 1 & 631.266 & 288.9319 & $0.0004^{*}$ \\
AB & 4.02 & 1 & 4.020 & 1.8400 & 0.2680 \\
AC & 42.25 & 1 & 42.250 & 19.3379 & $0.0218^{*}$ \\
BB & 102.52 & 1 & 102.516 & 46.9217 & $0.0064^{*}$ \\
BC & 264.06 & 1 & 264.063 & 120.8621 & $0.0016^{*}$ \\
CC & 36.48 & 1 & 36.482 & 16.6977 & 0.0265 \\
Falta de ajuste & 278.93 & 3 & 92.976 & 42.56 & $0.0059^{*}$ \\
Error total & 6.55 & 3 & 2.185 & & \\
Total (corr.) & 4228.14 & 15 & & & \\
\hline
\end{tabular}

$\mathrm{p}^{*}(<0.05)=$ estadísticamente significativo; $\mathrm{A}=$ temperatura, $\mathrm{B}=\mathrm{pH}, \mathrm{C}=$ concentración de cianuro libre $\left[\mathrm{CN}^{-}\right.$ ], $\mathrm{AA}=$ interacción entre las temperaturas, $\mathrm{AB}=$ interacción entre la temperatura y $\mathrm{pH}, \mathrm{AC}=$ interacción entre la temperatura y $\left[\mathrm{CN}^{-}\right], \mathrm{BB}=$ interacción entre los $\mathrm{pH}, \mathrm{BC}=$ interacción entre el $\mathrm{pH}$ y la $\left[\mathrm{CN}^{-}\right]$, y $\mathrm{CC}=$ interacción entre las $\left[\mathrm{CN}^{-}\right]$y $\left[\mathrm{CN}^{-}\right] . \mathrm{R}^{2}=93.25 \% ; \mathrm{R}^{2}$ (ajustada) $=83.12 \%$

Optimización mediante el diseño superficie respuesta

El efecto de los factores $\mathrm{B}, \mathrm{C}$ y las interacciones $\mathrm{AA}, \mathrm{AC}, \mathrm{BB}, \mathrm{BC}, \mathrm{CC}$ fueron significativos. Sin embargo, el factor A y la interacción $\mathrm{AB}$ no fueron significativas, por lo que se podría asumir que estas interacciones actuaron independientemente (Tabla 5). El valor de $\mathrm{R}^{2}$ ajustado fue $83.12 \%$, lo que implica que la variabilidad obtenida puede ser explicada correctamente mediante el modelo. Por lo tanto, el modelo puede ser empleado para realizar predicciones del proceso y optimizar los parámetros de operación. Según el ajuste del modelo de regresión, la ecuación polinómica fue:

$$
\begin{aligned}
& \%\left[\mathrm{CN}^{-}\right] \\
& =1615.66+33.202 \mathrm{~T}-395.263 p H \\
& +1.583\left[\mathrm{CN}^{-}\right]-0.503 \mathrm{~T}^{2}-0.401 \mathrm{TpH} \\
& +0.006 \mathrm{~T}\left[\mathrm{CN}^{-}\right]+20.25 \mathrm{TpH} \mathrm{H}^{2} \\
& -0.162 p H\left[\mathrm{CN}^{-}\right] \\
& +0.000\left[\mathrm{CN}^{-}\right]^{2}
\end{aligned}
$$

Donde:

$\%\left[\mathrm{CN}^{-}\right]=$Porcentaje de biodegradación de cianuro libre

$\mathrm{T}=$ Temperatura

$\left[\mathrm{CN}^{-}\right]=$Concentración de cianuro libre 
Tabla 6. Coeficiente de regresión para la eficiencia de biodegradación de cianuro

\begin{tabular}{cc}
\hline Coeficiente & Estimado \\
\hline constante & 1615.66 \\
A: T & 32.202 \\
B: $\mathrm{pH}$ & -395.263 \\
C: $[\mathrm{CN}-]$ & 1.583 \\
AA & -0.503 \\
AB & -0.401 \\
AC & 0.006 \\
BB & 20.25 \\
BC & -0.162 \\
CC & 0.000 \\
\hline
\end{tabular}

El diagrama de Pareto se determinó para descubrir las variables que afectan en la degradación (Fig. 2 ), que son las que tienen mayor influencia sobre la variable respuesta. Los factores significativos fueron el $\mathrm{pH}$, la concentración de $\mathrm{CN}-\mathrm{y}$ las interacciones AA, BC, BB, AC, CC (Fig. 2). En cambio, la temperatura, interacción AB resultaron ser las de menor efecto sobre la variable respuesta.

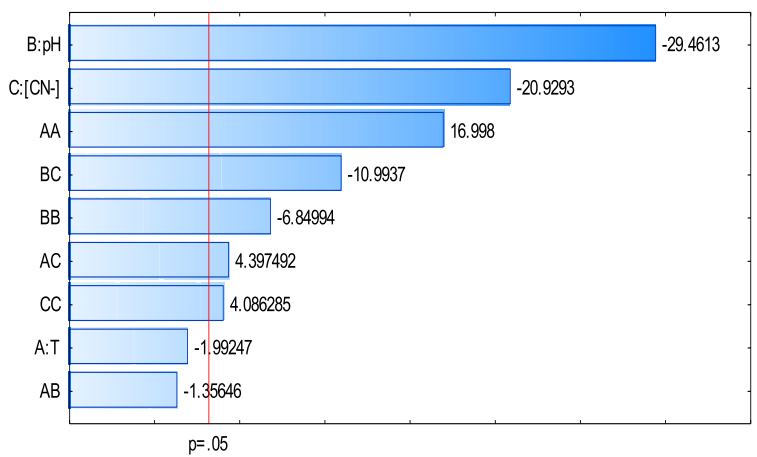

Figura 2. Diagrama de Pareto, selección de variables por diseño Box-Behnken. Los factores seleccionados deben tener un valor $\mathrm{p}<0.05$

Las condiciones óptimas de biodegradación de cianuro libre fueron a una temperatura de $30{ }^{\circ} \mathrm{C}$, $\mathrm{pH} 10$ y $250 \mathrm{ppm}\left[\mathrm{CN}^{-}\right]$. Según los valores predichos, la máxima remoción de cianuro fue de $99.06 \%$, con las variables experimentales a $30^{\circ} \mathrm{C}$, pH 10 y 200 ppm [CN'] (Tabla 7).

Tabla 7. Resultados estimados para la biodegradación de cianuro utilizando el modelo de Regresión Lineal

\begin{tabular}{|c|c|c|c|c|c|}
\hline \multirow{3}{*}{ TRAT. } & \multicolumn{3}{|c|}{ VARIABLE INDEPENDIENTE } & \multirow{2}{*}{\multicolumn{2}{|c|}{$\begin{array}{l}\text { VARIABLE. DEPENDIENTE } \\
\text { Eficiencia de remoción \% }\end{array}$}} \\
\hline & \multirow{2}{*}{ A: $\mathbf{T}\left({ }^{\circ} \mathrm{C}\right)$} & \multirow{2}{*}{ B: pH } & \multirow{2}{*}{$\begin{array}{c}\mathrm{C}:[\mathrm{CN}-] \text { inicial } \\
(\mathrm{ppm})\end{array}$} & & \\
\hline & & & & Observado & Predicho \\
\hline 1 & 25 & 10.0 & 300 & 83.33 & 92.66 \\
\hline 2 & 35 & 10.0 & 300 & 94.67 & 83.68 \\
\hline 3 & 25 & 11.0 & 300 & 52.00 & 83.71 \\
\hline 4 & 35 & 11.0 & 300 & 59.33 & 49.90 \\
\hline 5 & 25 & 10.5 & 200 & 86.00 & 89.77 \\
\hline 6 & 35 & 10.5 & 200 & 66.00 & 79.88 \\
\hline 7 & 25 & 10.5 & 400 & 56.00 & 96.16 \\
\hline 8 & 35 & 10.5 & 400 & 49.00 & 68.24 \\
\hline 9 & 30 & 10.0 & 200 & 98.00 & 99.06 \\
\hline 10 & 30 & 11.0 & 200 & 86.00 & 95.24 \\
\hline 11 & 30 & 10.0 & 400 & 94.00 & 78.29 \\
\hline 12 & 30 & 11.0 & 400 & 49.50 & 91.44 \\
\hline 13 & 30 & 10.5 & 300 & 79.33 & 83.29 \\
\hline 14 & 30 & 10.5 & 300 & 81.33 & 83.29 \\
\hline 15 & 30 & 10.5 & 300 & 78.00 & 83.29 \\
\hline 16 & 30 & 10.5 & 300 & 80.67 & 83.29 \\
\hline
\end{tabular}

\section{Interacción entre factores}

A medida que se incrementó el pH, disminuyó el porcentaje de biodegradación de cianuro por Klebsiella sp. ART1. A su vez, a medida que se incrementó la concentración de cianuro libre, se observó una disminución en la biodegradación de cianuro (Fig. 3). A medida que se incrementó la concentración de cianuro libre, disminuyó la biodegradación de cianuro; a su vez, a medida que se incrementó la temperatura se incrementó la biodegradación de cianuro (Fig. 4). A medida que se incrementó el $\mathrm{pH}$, disminuyó la biodegradación de cianuro y al incrementar la temperatura, aumentó la biodegradación (Fig. 5). 


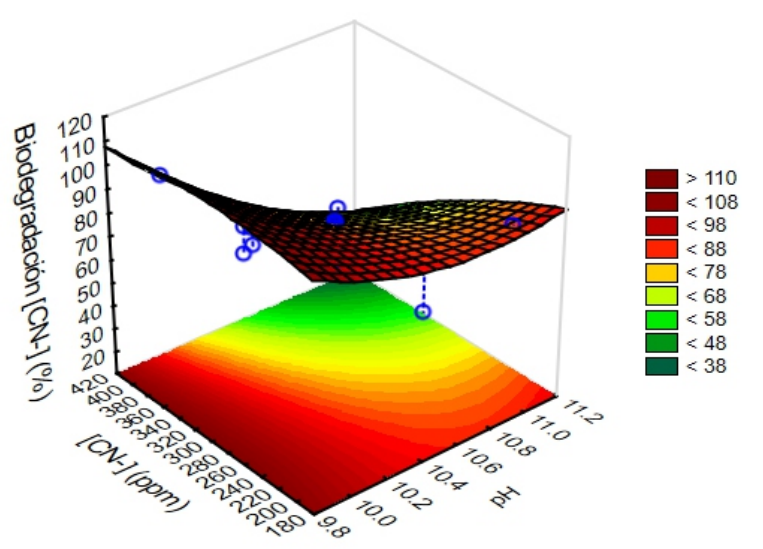

Figura 3. Gráfico de superficie respuesta de $\mathrm{pH}$ y concentración de cianuro libre con el porcentaje de cianuro biodegradado.

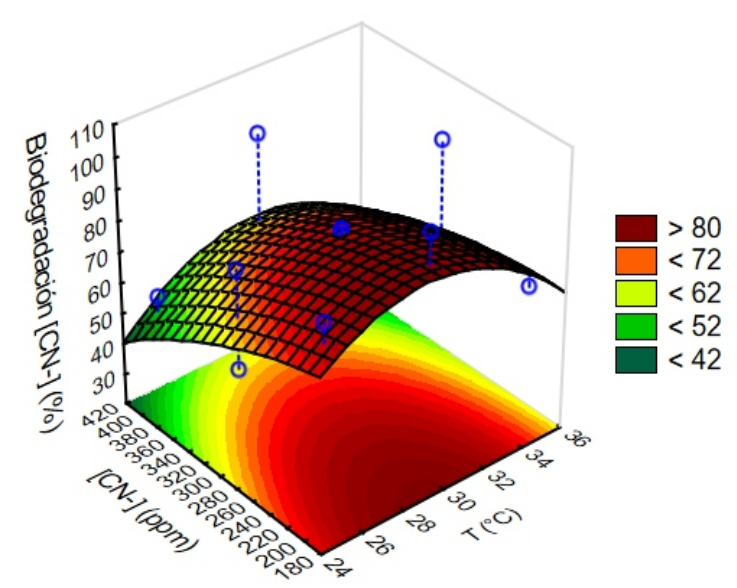

Figura 4. Gráfico de superficie respuesta de temperatura y concentración de cianuro con el porcentaje de cianuro biodegradado.

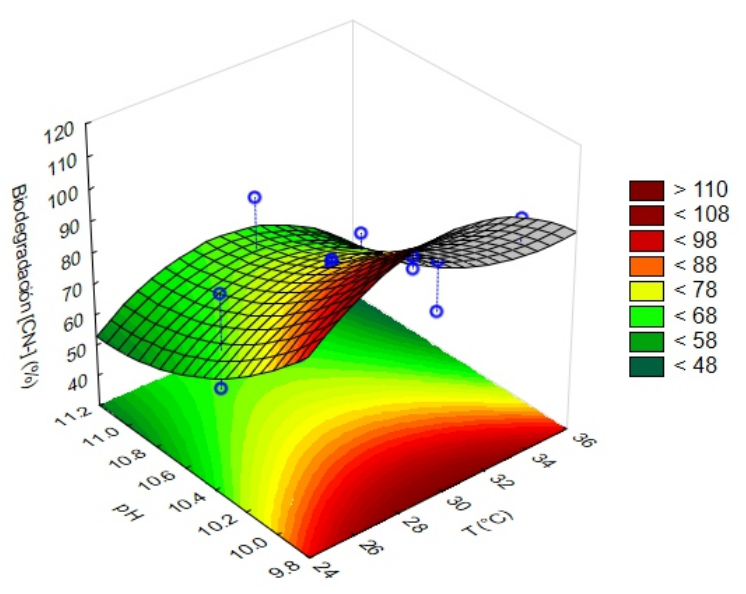

Figura 5. Gráfico de superficie respuesta de temperatura y $\mathrm{pH}$ con el porcentaje de cianuro biodegradado.
Las condiciones óptimas de biodegradación de cianuro libre fueron a una $30^{\circ} \mathrm{C}, \mathrm{pH} 10$ y $\left[\mathrm{CN}^{-}\right] \mathrm{de}$ $250 \mathrm{ppm}$.

\section{DISCUSIÓN}

\section{Crecimiento de la bacteria en presencia de cianuro}

De acuerdo a la caracterización y pruebas bioquímicas realizadas, estas indican que la cepa corresponde al género Klebsiella sp. (Garrity et al., 2010). La capacidad de crecimiento de Klebsiella sp. ART1 fue debido a que tuvo la capacidad de degradar cianuro y pudo utilizarlo como única fuente de nitrógeno, similar a lo descrito por Kao et al. (2003) y Chen et al. (2008), quienes indican que Klebsiella oxytoca puede utilizar KCN como la única fuente de nitrógeno. Además, se ha utilizado para la degradación hidrocarburos, tetracianoniquelato y nitrilo (Kao et al., 2003; Chen et al., 2009; Liu et al., 2017). Por lo tanto, tiene un alto potencial para ser utilizado en el tratamiento de aguas residuales que contienen cianuro (Chen et al., 2009).

En este estudio probablemente Klebsiella sp. utilizó la ruta hidrolítica, mediante la enzima nitrilo hidratasa o nitrilasas, que posteriormente lo convierten a amonio, metano, $\mathrm{CO}_{2}$, ácido fórmico y/o ácido carboxílico (Gupta et al., 2010; LuqueAlmagro et al., 2018), debido a que no se utilizó ninguna fuente adicional de nitrógeno.

Desde el punto de vista químico, el tratamiento biológico de efluentes cianurados requiere un $\mathrm{pH}$ alcalino (Das \& Santra, 2011). Sin embargo, estudios realizados por L Lovasoa et al., (2017) Chen et al. (2009); y Kao et al. (2006) indican que las condiciones óptimas de biodegradación de cianuro libre por Klebsiella sp. son en $\mathrm{pH}$ neutros. No obstante, Suzuki et al. (2014) indica que también tiene la capacidad de crecer en condiciones alcalinas.

\section{Determinación de la eficiencia de degradación de cianuro}

La cepa de Klebsiella sp. ART1 tuvo la capacidad de tolerar y degradar 400 ppm de cianuro libre en condiciones alcalinas; obteniéndose la degradación de cianuro y producción de amonio, este proceso fue similar al descrito por (Kao et al., 2003), que indica que la biodegradación de cianuro y la producción de amonio ocurre simultáneamente. Según la presente 
investigación, podemos afirmar que mientras la concentración de cianuro disminuye la producción de amonio aumenta. Otros estudios, realizados en el mismo género, indican que Klebsiella oxytoca tiene la capacidad de tolerar y degradar cianuro (Chen at al., 2008), Klebsiella pneumoniae tuvo la capacidad de degradar tiocianato entre 500 a $2500 \mathrm{ppm}$ a un $\mathrm{pH}$ de $6.0 \mathrm{y}$ $37^{\circ} \mathrm{C}$ (Chaudhari \& Kodam, 2010); sin embargo, ambos procesos de degradación se realizaron en condiciones neutras.

Existen varios microorganismos implicados en el proceso de biodegradación de cianuro como Pseudomonas fluorescens, que obtuvo una eficiencia de degradación del $85 \%$ de $\mathrm{KCN}$ en $6 \mathrm{~h}$; el metabolismo de esta bacteria es dependiente de oxígeno, además de haber presencia de $\mathrm{CO}_{2}$ y amoníaco como productos finales de la biodegradación (Kunz et al., 1992). Asimismo, Pseudomonas aeruginosa STK 03 tuvo una eficiencia de degradación de cianuro libre de 80 y $32 \%$, a partir de una concentración inicial de 250 y $450 \mathrm{mg} \mathrm{CN}^{-} / \mathrm{L}$ respectivamente. Estos procesos de degradación se realizaron en condiciones alcalinas (Mekuto et al., 2016); mientras que Pseudomonas pseudoalcaligenes W2 fue capaz de tolerar una concentración de cianuro de 39 $\mathrm{mg} / \mathrm{L}$ (Tiong et al. 2015); Azotobacter vinelandii degradó un $84.4 \%$ de cianuro con $3 \mathrm{mM}$ de $\mathrm{NaCN}$ (Sarawut et al., 2013) y Bacillus tuvo una máxima degradación de cianuro de $700 \mathrm{mg} / \mathrm{L}$ (Wu et al., 2014). Otras investigaciones realizadas por Razanamahandry et al., (2016) probaron la capacidad de degradar de cianuro libre a un $\mathrm{pH}$ 9.5 , donde se obtuvo una degradación del $95 \%$ en $25 \mathrm{~h}$. Todos estos estudios confirman nuestros resultados y reafirman que esta cepa tiene un alto potencial de ser utilizada en procesos de biodegradación de cianuro debido a que obtuvo una eficiencia de degradación del $98 \%$, en un total de $60 \mathrm{~h}$ en condiciones alcalinas. Es importante considerar que la degradación se asoció con el crecimiento bacteriano y la producción de amonio. Sin embargo, concentraciones elevadas de cianuro pueden inhibir el crecimiento bacteriano y afectar la degradación de cianuro.

El proceso de biodegradación de cianuro se produce en condiciones ácidas, neutras y ligeramente alcalinas (Razanamahandry et al., 2016). Sin embargo, Hande \& Seyis (2016) recomiendan trabajar en condiciones alcalinas, para evitar la volatilización de cianuro en forma de ácido cianhídrico en condiciones de $\mathrm{pH}$ neutras y ácidas — (Eisler \& Wiemeyer, 2004; Kumar et al., 2018; Luque-Almagro et al., 2016; Razanamahandry et al., 2018). Por lo tanto, en este tipo de procesos, es importante considerar el crecimiento de la bacteria en condiciones alcalinas.

\section{Optimización mediante diseño superficie respuesta}

El proceso de biodegradación de cianuro por Klebsiella sp. ART1 se optimizó utilizando el método de superficie respuesta con la finalidad de determinar la mejor condición para degradar este compuesto. Para ello, se consideró tres parámetros: la temperatura, $\mathrm{pH}$ y concentración de cianuro.

Los resultados de optimización por Klebsiella sp. ART1, en condiciones alcalinas, tuvieron una eficiencia de degradación de cianuro libre de $99.06 \%$ a una temperatura de $30^{\circ} \mathrm{C}, \mathrm{pH}$ de $10 \mathrm{y}$ una concentración de $\left[\mathrm{CN}^{-}\right]$de $250 \mathrm{ppm}$, similares a las investigaciones realizadas por Moradkhani et al., (2018), en la que Pseudomonas parafulva tuvo una mayor eficiencia de degradación de cianuro $(93.5 \%)$ cuando la concentración inicial era de $200 \mathrm{ppm}$. Sin embargo, es importante considerar que concentraciones elevadas de cianuro tienen un efecto destructivo para los microorganismos que tienen la capacidad de eliminar cianuro, esto significa que el cianuro era utilizado como sustrato hasta $200 \mathrm{ppm}$, pero en concentraciones elevadas presentaba un efecto inhibidor de crecimiento. Entonces, al aumentar la concentración de cianuro, disminuye la eficiencia de degradación o el tiempo requerido para la degradación aumenta notablemente. Otra cepa bacteriana tuvo la capacidad de tolerar 500 ppm de cianuro libre. En este caso, la degradación del cianuro se relacionó con el crecimiento bacteriano y alcanzó un nivel máximo del $96 \%$ de degradación durante la fase exponencial; en la cual, la tasa de crecimiento más alta fue de $1.23 \times 10^{8}$ cél $/ \mathrm{ml}$ ) en 4 días de incubación. Entonces, podemos afirmar que mientras que la carga microbiana aumenta también aumenta la biodegradación de cianuro, y que el crecimiento bacteriano y la eliminación de cianuro están correlacionados (Mirizadeh et al., 2014).

La optimización del proceso de biodegradación se ha realizado en Bacillus sp., Serratia marcense, entre otros (Javaheri et al., 2017, Karamba et al., 2015; Kumar et al., 2015; Mekuto et al., 2015; Mieizadeh et al., 2014, Moradkhari et al., 2018), pero la optimización de degradación de cianuro 
mediada por de Klebsiella en condiciones alcalinas aún no se ha reportado, a excepción de la investigación realizada por Hande \& Seyis (2016), que ha optimizado la degradación de cianuro por Klebsiella pneumoniae a un $\mathrm{pH}$ neutro.

En una investigación realizada por Javaheri et al., (2017), se indica que Bacillus sp. logra un $86 \%$ de degradación cuando la concentración de $\mathrm{CN}^{-}$fue $200 \mathrm{mg} / 1$ y que al reducir la concentración de cianuro libre se logra una mayor degradación (Wu et al. 2014). Igualmente para Pseudomonas putida, que crece en una concentración de cianuro $4 \mathrm{mM}$ y se inhibió a $8 \mathrm{mM}$ (Chapatwala et al. 1998). Otra investigación indica que la optimización en condiciones alcalinas fue a una temperatura de $34.2{ }^{\circ} \mathrm{C}, \mathrm{pH} 10.3$ y concentración de glucosa $0.44(\mathrm{~g} / \mathrm{L})$, con una concentración de 500 ppm de cianuro (Mirizadeh et al., 2014).

Teniendo en cuenta la concentración de cianuro y el tiempo, la degradación de $200 \mathrm{mg} / \mathrm{L}$ se efectuó en 90 horas con una concentración inicial de 400 ppm, similar a la optimización realizada por Mekuto et al., (2015), en la que las condiciones favorables fueron a $33.60{ }^{\circ} \mathrm{C}, \mathrm{pH} 9.88$ de 206.53 $\mathrm{mg} / \mathrm{l}$ en 96 h, a partir de una concentración inicial de cianuro de $500 \mathrm{mg} \mathrm{CN}^{-} / \mathrm{L}$. En el caso de Serratia marcescens, se observó que la bacteria degradaba el $95.6 \%$ de $200 \mathrm{mg} / \mathrm{L}$ de $\mathrm{KCN}$ en las condiciones optimizadas, de $\mathrm{pH} 6$, temperatura 32.5 , tamaño de inóculo $20 \%$; y se informa que las bacterias degradan el cianuro en amoníaco, formamida o formiato y dióxido de carbono, subproductos menos tóxicos (Karamba et al., 2016). De acuerdo a nuestra investigación y otras realizadas en biodegradación, se observó que un el umbral máximo de biodegradación de cianuro era de 200 mg/L (Kuyucak \& Akcil, 2013), pero en procesos similares debemos tener en cuenta la investigación realizara por Kao et al. (2003), que informa que una concentración de cianuro superior a $2.6 \mathrm{mg} / \mathrm{l}$ puede inducir una fase de retraso en el crecimiento bacteriano, y esto afecta en el proceso de biodegradación de cianuro, por lo que es importante determinar la tolerancia de los microorganismos antes de realizar la biodegradación de cianuro.

\section{CONCLUSIONES}

La cepa de Klebsiella sp. ART1 ha sido optimizada para la degradación de cianuro. Los valores óptimos fueron a $\mathrm{pH} 10$, a una temperatura de $30{ }^{\circ} \mathrm{C}$ y con una concentración de $250 \mathrm{ppm}$ de cianuro libre, según el diseño de Box- Behnken; acuerdo a estas condiciones, se obtuvo una eficiencia de degradación de un $99 \%$ en 60 horas. Con el desarrollo bacteriano se generó como producto final hasta $1.0 \mathrm{mg} / \mathrm{L}$ de amonio. Esto sugiere que la bacteria podría ser utilizada como una alternativa de tratamiento en procesos de biorremediación.

\section{REFERENCIAS}

Akcil, A. (2003). Destruction of cyanide in gold mill effluents: Biological versus chemical treatments. Biotechnology Advances, 21(6), 501-511. doi: $10.1016 / \mathrm{S} 0734$ 9750(03)00099-5

Akinpelu, E. A., Adetunji, A. T., Ntwampe, S. K. O., Nchu, F., \& Mekuto, L. (2017). Biochemical characteristics of a free cyanide and total nitrogen assimilating Fusarium oxysporum EKT01/02 isolate from cyanide contaminated soil. Data in B rief, $\quad 14, \quad 84-87$. doi:10.1016/j.dib.2017.07.023

Box GEP, Behnken DW. (1960). Some New Three Level Designs for the Study of Quantitative Variables. Taylor \& Francis Group, Ltd. On behalf of american statistical Association and American Society for Quality. Technometrics. 1960;2(4):455-75. http://www.jstor.org/stable/1266454.

Carrillo P., F. (2000). "Estudio cinético de la oxidación de cianuro y tiocianato con ozono." Centro de investigación y de estudios avanzados del Instituto Politécnico Nacional Unidad Saltillo.

Chapatwala, K. D., Babu, G. R. V., Vijaya, O. K., Kumar, K. P., \& Wolfram, J. H. (1998). Biodegradation of cyanides, cyanates and thiocyanates to ammonia and carbon dioxide by immobilized cells of Pseudomonas putida. Journal of Industrial Microbiology and Biotechnology, 20(1), 28-33. doi: 10.1038/sj.jim.2900469

Chaudhari, A. U., \& Kodam, K. M. (2010). Biodegradation of thiocyanate using coculture of Klebsiella pneumoniae and Ralstonia sp. Applied Microbiology and Biotechnology, 85(4), 1167-1174. doi: 10.1007/s00253-009-2299-7

Chen L. Z.; Nguang S. K.; Chen X. D. (2005). Modeling and Optmization of Biotechnological Processes.

Chen, C. Y., Kao, C. M., \& Chen, S. C. (2008). Application of Klebsiella oxytoca 
immobilized cells on the treatment of cyanide wastewater. Chemosphere, 71(1), $133-139$.

doi:10.1016/j.chemosphere.2007.10.058

Chen, C. Y., Kao, C. M., Chen, S. C., \& Chen, T. Y. $\left(\begin{array}{llll}2 & 0 & 0 & 9\end{array}\right)$. B i o d e g r a d a tion of tetracyanonickelate by Klebsiella oxytoca under anaerobic conditions. Desalination, 249 ( 3 ), $1212-1216$. d o i : 10.1016/j.desal.2009.06.036

Das S., \& Santra S.C. (2011). Cyanice degradation by Aspergillus niger strain isolated from steel-plant wastewater. EletronicJournal of Environmental, Agricultural, and Food Chemistry 10(7):2516-2522.

Dash, R. R., Gaur, A., \& Balomajumder, C. (2009). Cyanide in industrial wastewaters and its removal: A review on biotreatment. Journal of Hazardous Materials, 163(1), 1-11. doi: 10.1016/j.jhazmat.2008.06.051

Dursun, A. Y. (2000). Biodegradation kinetics of ferrous (II) cyanide complex ions by immobilized Pseudomonas fluorescens in a packed bed column reactor. 35, 615-622.

Eisler, R., \& Wiemeyer, S. N. (2004). Cyanide hazards to plants and animals from gold mining and related water issues. Reviews of Environmental Contamination and Toxicology, 183 (1), 21-54. doi:10.1201/9781420037982-15

Erick, M. V. Z., Fernandez, C., Gerardo, M. C. C., \& Sosa, C. (2012). Bacteriologia $Y$ Micologia Veterinaria.

Garrity, G., Bell, J., \& Lilburn, T. (2010). Bergey's Manual of Systematic Bacteriology, vol $2 B$. In D. Brenner, N. Krieg, \& J. Staley (Eds.), editorial board (second edi). doi: 10.1245/s10434-010-1229-3

Gessner, T. P., Kadlec, R. H., \& Reaves, R. P. (2005). Wetland remediation of cyanide and hydrocarbons. Ecological Engineering, 25(4), 457-469. doi:10.1016/j.ecoleng.2005.07.015

Gupta, N., Balomajumder, C., \& Agarwal, V. K. (2010). Enzymatic mechanism and biochemistry for cyanide degradation: A review. Journal of Hazardous Materials, $176(1-3), \quad 1-13$. d o i : 10.1016/j.jhazmat.2009.11.038

Hande, N., \& Seyis, I. (2016). Biological treatment of cyanide by using Klebsiella pneumoniae species. Food Technology and Biotechnology, 54(4), 450-454. doi: 10.17113/ftb.54.04.16.4518
Huertas, M. J., Sáez, L. P., Roldán, M. D., LuqueAlmagro, V. M., Martínez-Luque, M., Blasco, R., García-García, I. (2010). Alkaline cyanide degradation by Pseudomonas pseudoalcaligenes CECT5344 in a batch reactor. Influence of pH. Journal of Hazardous Materials, $179(1-3), 72-78$. d o i : 10.1016/j.jhazmat.2010.02.059

Javaheri Safa, Z., Aminzadeh, S., Zamani, M., \& Motallebi, M. (2017). Significant increase in cyanide degradation by Bacillus sp. M01 PTCC 1908 with response surface methodology optimization. AMB Express, 7(1). doi: 10.1186/s13568-017-0502-2

Johnson, A. T. (2013). Teaching the principle of biological optimization. Journal of Biological Engineering. 1-7.

Kandasamy, S., Dananjeyan, B., Krishnamurthy, K., \& Benckiser, G. (2015). Aerobic cyanide degradation by bacterial isolates from cassava factory wastewater. Brazilian Journal of Microbiology, 46(3), 659-666. doi: 10.1590/S1517-838246320130516

Kao, C. M., Liu, J. K., Lou, H. R., Lin, C. S., \& Chen, S. C. (2003). Biotransformation of cyanide to methane and ammonia by Klebsiella oxytoca. Chemosphere, 50(8), 1055-1061. doi: $10.1016 / \mathrm{S} 0045$ 6535(02)00624-0

Kao, C. M., Chen, K. F., Liu, J. K., Chou, S. M., \& Chen, S. C. (2006). Enzymatic degradation of nitriles by Klebsiella oxytoca. Applied Microbiology and Biotechnology, 71(2), 228-233. doi: 10.1007/s00253-005-0129-0

Karamba, K. I., Yasid, N. A., \& Khalid, A. (2015). Isolation, screening and characterisation of cyanide-degrading Serratia marcescens strain aq07.

Khamar, Z., Makhdoumi-Kakhki, A., \& Mahmudy Gharaie, M. H. (2015). Remediation of cyanide from the gold mine tailing pond by a novel bacterial co-culture. International Biodeterioration and Biodegradation, 99, 123-128. doi: 10.1016/j.ibiod.2015.01.009

Kunz, D. A., Nagappan, O., Silva-avalos, J., \& Delongt, G. T. (1992). Utilization of Cyanide as a Nitrogenous Substrate by Pseudomonas fluorescens NCIMB 11764: Evidence for Multiple Pathways of Metabolic Conversion. 58(6), 2022-2029.

Kumar, V., Kumar, V., \& Bhalla, T. C. (2015). Statistical Enhancement of Cyanide Degradation Using Microbial Consortium. 
Journal of Microbial \& Biochemical Technology, 07(06), 344-350. doi: 10.4172/1948-5948.1000237

Kumar, R., Saha, S., Dhaka, S., Kurade, M. B., Kang, C. U., Baek, S. H., \& Jeon, B.-H. (2017). Remediation of cyanidecontaminated environments through microbes and plants: a review of current knowledge and future perspectives. Geosystem Engineering, 20(1), 28-40. doi: 10.1080/12269328.2016.1218303

Kumar, V., Kumar, V., \& Bhalla, T. C. (2018). Alkaline active cyanide dihydratase of Flavobacterium indicum MTCC 6936: Growth optimization, purification, characterization and in silico analysis. International Journal of Biological Macromolecules, 116(2017), 591-598. doi: 10.1016/j.ijbiomac.2018.05.075

Kuyucak, N., \& Akcil, A. (2013). Cyanide and removal options from effluents in gold mining and metallurgical processes. Minerals Engineering, 50-51, 13-29. doi: 10.1016/j.mineng.2013.05.027

Javaheri Safa, Z., Aminzadeh, S., Zamani, M., \& Motallebi, M. (2017). Significant increase in cyanide degradation by Bacillus sp. M01 PTCC 1908 with response surface methodology optimization. AMB Express, 7(1). doi: 10.1186/s13568-017-0502-2

Liu, C., Yuan, K., Chen, R. ping, Chen, M. jun, \& Yu, L. (2017). Biodegradation kinetics of nitriles with easily degradable substrate by Klebsiella oxytoca GS-4-08. International Biodeterioration and Biodegradation, 118, 95-101.doi: 10.1016/j.ibiod.2017.01.028

Lottermoser, B. (2010). Mine Wastes. Third ed.doi:10.1007/978-3-642-12419-8

Lovasoa, C. R., Hela, K., Harinaivo, A. A., \& Hamma, Y. (2017). Bioremediation of soil and water polluted by cyanide: A review. African Journal of Environmental Science and Technology, 11(6), 272-291. doi: 10.5897/AJEST2016.2264

Luque-Almagro, V. M., Blasco, R., MartínezLuque, M., Moreno-Vivián, C., Castillo, F., \& Roldán, M. D. (2011). Bacterial cyanide degradation is under review: Pseudomonas pseudoalcaligenes CECT5344, a case of an alkaliphilic cyanotroph. Biochemical Society Transactions, 39(1), 269-274. doi: 10.1042/BST0390269

Luque-Almagro, V. M., Moreno-Vivián, C., \& Roldán, M. D. (2016). Biodegradation of cyanide wastes from mining and jewellery industries. Current Opinion in Biotechnology, 38, 9-13. doi:10.1016/j.copbio.2015.12.004

Luque-Almagro, V. M., Cabello, P., Sáez, L. P., Olaya-Abril, A., Moreno-Vivián, C., \& Roldán, M. D. (2018). Exploring anaerobic environments for cyanide and cyanoderivatives microbial degradation. Applied Microbiology and Biotechnology, 102(3), 1067-1074. doi: 10.007/s00253-017-86786

Mekuto, L., Ntwampe, S. K. O., \& Jackson, V. A. (2015). Biodegradation of free cyanide and subsequent utilisation of biodegradation byproducts by Bacillus consortia: Optimisation using response surface methodology. Environmental Science and Pollution Research, 22(14), 10434-10443. doi: 10.1007/s11356-015-4221-4

Mekuto, L., Ntwampe, S. K. O., Kena, M., Golela, M. T., \& Amodu, O. S. (2016). Free cyanide and thiocyanate biodegradation by Pseudomonas aeruginosa STK 03 capable of heterotrophic nitrification under alkaline conditions. 3 Biotech, 6(1), 1-7. doi: 10.1007/s13205-015-0317-2

Mirizadeh, S., Yaghmaei, S., \& Ghobadi Nejad, Z. (2014). Biodegradation of cyanide by a new isolated strain under alkaline conditions and optimization by response surface methodology (RSM). Journal of Environmental Health Science \& Engineering, 12(1), 85. doi: 10.1186/2052336X-12-85

Mondal, P., Balomajumder, C., \& Dwivedi, N. (2017). Bioremoval of cyanide from aqueous solution using Tectona grandis leaves powder: a potential bioadsorbent. International Journal of Environmental Technology and Management, 19(3/4), 198. doi:10.1504/ijetm.2016.10003103

Mongomery, D. (2004). Diseño y Análisis de Experimentos. (L. Wiley, Ed.) (segunda ed). New York: universidad estatal de Arizona.

Moradkhani, M., Yaghmaei, S., \& Nejad, Z. G. (2018). Biodegradation of cyanide under alkaline conditions by a strain of Pseudomonas putida isolated from gold mine soil and optimization of process variables through response surface methodology (RSM). Periodica Polytechnica Chemical Engineering, 62(3), 265-273. doi: 10.3311/PPch.10860

Nyamunda, B. C. (2017). Review of the Impact on 
Water Quality and Treatment Options of Cyanide Used in Gold Ore Processing. W a t e r $Q$ u a lity. http://doi.org/10.5772/65706

Ram, A. V. (2010). Toxicidad del cianuro. Investigación bibliográfica de sus efectos en animales y en el hombre. Revista de Medicina Del Perú, 71(1), 54-61. doi:10.1016/S0166-445X(96)00815-6

Razanamahandry, L. C., Andrianisa, H. A., Karoui, H., Kouakou, K. M., \& Yacouba, H. (2016). Biodegradation of free cyanide by bacterial species isolated from cyanidecontaminated artisanal gold mining catchment area in Burkina Faso. Chemosphere, 157, 71-78. doi: 10.1016/j.chemosphere.2016.05.020

Sarawat K., S., Vichitphan, S., Laopaiboon, L., Vichitphan, K., \& Han, J. (2013). Growth and cyanide degradation of Azotobacter vinelandii in cyanide-containing wastewater system. Journal of Microbiology and Biotechnology, 23(4),
572-578. doi: 10.4014/jmb.1209.09026

Suzuki, T., Nishikawa, C., Seta, K., Shigeno, T., \& Nakajima-Kambe, T. (2014). Ethanol production from glycerol-containing biodiesel waste by Klebsiella variicola shows maximum productivity under alkaline conditions. New Biotechnology, $31(3), \quad 246-253$. doi:10.1016/j.nbt.2014.03.005

Tiong, B., Bahari, Z. M., Irwan Shah Lee, N. S., Jaafar, J., Ibrahim, Z., \& Shahir, S. (2015). Cyanide degradation by Pseudomonas pseudoalcaligenes strain W2 isolated from mining effluent. Sains Malaysiana, 44(2), 233-238. doi: 10.17576/jsm-2015-4402-10

Wu, C. F., Xu, X. M., Zhu, Q., Deng, M. C., Feng, L., Peng, J., ... Wang, J. H. (2014). An effective method for the detoxification of cyanide-rich wastewater by Bacillus sp. CN-22. Applied Microbiology and Biotechnology, 98(8), 3801-3807. doi: 10.1007/s00253-013-5433-5 